\title{
Les formations du secteur aménagement : genèse et évolutions
}

Les disciplines en questions. Dans Hervé, N. et Gardies, C (dir.) Dijon, France : éducagri, Collection recherche Agora. Bouillier M.H., O. Bories, Asloum N., (2015).

\section{Résumé}

Dans ce texte nous présentons la création, au début des années 1990, d'un secteur de formation «aménagement» au sein de l'enseignement technique agricole. Nous présentons l'origine de ce choix politique à partir d'une approche historique du contexte social du début des années 1990, marqué par la montée en puissance d'une demande sociale de qualité de l'environnement, et la façon dont ces évolutions sociales ont été traduites au sein des prescriptions des différents diplômes qui composent le secteur.

Dans une seconde partie nous analysons les changements apportés par les dernières réformes, en particulier la réforme de la voie professionnelle.

Mots clés : Aménagement, formation professionnelle initiale, prescriptions, curricula, développement durable.

La perspective donnée à cet ouvrage nous invite à analyser l'origine et les caractéristiques d'un secteur de formation créé récemment dans l'enseignement technique agricole: le secteur aménagement.

Il rassemble des formations anciennes conduisant à des métiers connus comme ceux liés à la gestion forestière ou à l'aménagement des jardins, et de nouvelles formations préparant à des métiers émergeants ou à de nouvelles fonctions attribuées à l'espace rural considéré comme un espace de nature par la société. Ce nouveau secteur constitue l'une des réponses apportées par l'enseignement agricole aux attentes sociales croissantes en matière de préservation de l'environnement.

L'étude de cette création présente selon nous plusieurs intérêts.

Un intérêt sociologique d'abord, en offrant la possibilité d'analyser le processus par lequel un système éducatif traduit un ensemble de changements sociaux, technologiques et professionnels en contenus d'enseignement. Notre texte s'appuie sur des travaux de recherche que nous avons conduits au moment de la création de ce secteur et qui se réfère au courant de pensée de "la nouvelle sociologie de l'éducation » qui s'est développé en Angleterre au cours des années 1970 et en France à partir de la traduction qu'en ont fait Trottier (1987) et Forquin (1992). Cette sociologie, fondée sur l'hypothèse du caractère socialement construit des savoirs scolaires, postule que l'école n'est pas le simple reflet des évolutions sociales et culturelles mais qu'elle sélectionne ce qui doit faire l'objet d'un apprentissage institutionnalisé.

Cette étude présente également un intérêt épistémologique, car elle propose une analyse des concepts et des démarches pédagogiques mobilisées pour assurer cette traduction alors qu'il s'agit de métiers émergeants pour lesquels il n'existe pas encore de corpus scientifique et technique stabilisés. 
Cette situation permet de prendre conscience de la fonction sociale remplie par les disciplines qui traduisent un certain regard sur le monde (Fourez, 1996).

Enfin, ce secteur de formation occupe une place particulière vis-à-vis du champ plus général de l'éducation à l'environnement qui a commencé à se développer à la fin des années 1980. Il fait selon nous figure de pionnier en proposant des contenus très novateurs. Son originalité se situe principalement dans les visées qui le caractérisent. Il ne s'agit pas, dans ces filières professionnelles, uniquement d'éduquer à une nouvelle vision du monde et de la nature mais également de former à agir sur elle de manière responsable dans le cadre d'un métier qui exige d'obtenir des résultats acceptables socialement et économiquement. Cette exigence a conduit les concepteurs des référentiels à formuler des propositions plus précises et plus concrètes que celles qui caractérisent généralement l'éducation à l'environnement.

Ce texte s'organise en deux grandes parties.

Une première partie présente la genèse du secteur aménagement : le contexte socioéconomique et institutionnel qui a présidé à sa mise en place et la façon dont l'institution a répondu aux évolutions de contexte à travers les prescriptions qu'elle affiche. Nous présenterons le cadre d'analyse des prescriptions utilisé ainsi que des éléments d'interprétation des choix affichés à partir de résultats de travaux de recherche (Baradat-Bouillier, 1999).

La deuxième partie s'intéresse aux évolutions apportées par les réformes et rénovations actuelles, au regard notamment de deux dynamiques : le renouvellement de l'approche professionnelle des cursus, et le développement pris par l'éducation au développement durable aujourd'hui dans l'ensemble des formations.

\section{La genèse du secteur aménagement}

On peut considérer que la création d'un secteur aménagement dans l'enseignement technique agricole s'inscrit dans un débat engagé dès les années 1980. L'année 1985, avec la création du Brevet de Technicien Agricole (BTA), marque le début d'une réforme qui a posé les bases d'une structuration originale des programmes de l'enseignement technique agricole.

Entre 1985 et 1987 s'engage des réflexions sur la poursuite de cette rénovation à l'ensemble des diplômes. C'est dans ce cadre qu'une commission du Conseil Général d'Agronomie « qualifications professionnelles et enseignement agricole », composée de plusieurs groupes thématiques, est mise en place.

Le rapport qu'elle produit en 1987 à propos des filières de formation et des qualifications à développer dans l'enseignement technique agricole constitue pour nous un premier texte de référence.

\section{I.1. Le contexte social, politique et économique dans lequel ce secteur a été créé}

\section{I.1.1. Les territoires ruraux : un réservoir d'emploi}


La commission «qualification »propose la formation de techniciens en aménagement à partir d'un argumentaire principalement centré sur des questions d'aménagement du territoire. L'émergence de nouveaux emplois et de nouvelles qualifications apparaît alors nécessaire face à un contexte nouveau ${ }^{1}$ :

- contraste de plus en plus accentué entre zones de haute productivité agricole et zones exclues de cette économie de marché et par conséquent menacées d'abandon ;

- pression de l'habitat en zone périurbaine ;

- pression de fréquentation sur l'ensemble du territoire en fonction d'une demande liée au tourisme et aux loisirs beaucoup plus diffuse.

Le tourisme est présenté comme l'une des voies du développement rural, en générant des demandes en matière d'équipements, de gestion d'espaces (qualité du cadre de vie, sécurité, prévention des risques, etc.), d'animation et d'une manière générale d'organisation de tous les services et prestations associés.

Cet ensemble de mutations est mis en perspective dans un contexte de décentralisation. Les communes sont censées s'entourer de nouvelles compétences pour mettre en œuvre et gérer des projets locaux.

\section{I.1.2. Une réponse aux préoccupations environnementales de la société}

L'année 1990 est signalée par de nombreux auteurs comme un tournant dans les relations entre agriculture et environnement. Elle est marquée par une vive querelle qui opposa les ministères de l'environnement et de l'agriculture à propos de l'application du principe «pollueur-payeur » aux agriculteurs jugés responsables de la pollution des nappes phréatiques par les nitrates.

Le plan national pour l'environnement publié cette même année dresse alors un bilan médiocre de l'état de notre environnement, n'épargnant pas l'agriculture. La question des nuisances provoquées par les activités agricoles n'est pas nouvelle, elle a jalonné la modernisation de l'agriculture. Ce qui est nouveau et que révèle cette politisation du débat, c'est à la fois la montée en puissance dans la société française des préoccupations concernant la qualité de l'environnement, et surtout l'entrée de l'activité agricole dans son ensemble, au même titre que d'autres activités industrielles, dans le champ des problèmes d'environnement devant faire l'objet d'une régulation sociale.

Le début des années 1990 est également marqué par la réforme de la politique agricole commune. Impératifs écologiques et économiques convergent pour définir une réorientation de notre agriculture. La préservation de l'environnement devient une des dimensions de la politique agricole à travers les mesures agri-environnementales. C'est à partir de ce moment-là que le terme d'environnement est utilisé pour définir les orientations de ce secteur.

Le schéma prévisionnel national (1991-1993) est pour nous un second texte de référence. Il fait de la prise en compte des préoccupations de qualité des produits et de l'environnement sa deuxième priorité, après la formation au niveau IV des futurs chefs d'exploitation. Deux grands axes sont définis : limiter les pollutions liées à la production agricole, et assurer la survie de l'espace rural. Il préconise l'élargissement du champ couvert par les formations qui doit aller «jusqu'à la prise en main de la gestion de notre environnement et de sa protection. L'enseignement agricole, avec sa composante biologique forte est le mieux placé pour développer les approches écologiques et

\footnotetext{
${ }^{1}$ Nous reprenons ici les principaux axes de l'analyse présentée dans ce texte.
} 
économiques permettant de valoriser notre environnement ». Ce texte invite à réfléchir à la création de «nouvelles formations aux métiers de l'aménagement et de l'environnement, à côté de la rénovation des formations liées à la création et l'entretien des parcs et jardins, d'espaces de loisirs et de sports, de même que les formations liées à l'exploitation et à la valorisation des forêts ».

Il fait écho en cela :

- à l'analyse présentée dans le plan national pour l'environnement, qui fait de la formation un élément fort de la politique de ce secteur, en proposant de former des corps spécifiques de techniciens et un corps d'inspecteurs de l'environnement,

- et à celle du commissariat général du plan (rapport sur le plan national de l'environnement d'octobre 1990) qui note l'avènement probable de nombreux métiers de l'environnement notamment pour l'écologie urbaine (génie de l'environnement), pour la découverte des milieux naturels (animateurs), pour l'entretien, le gardiennage, la gestion des milieux naturels (génie écologique). Ce rapport recommande la création de formations pluridisciplinaires de type bac +5 , bac +2 , la création de filières spécifiques «environnement » du brevet d'études professionnelles agricole (BEPA) au Brevet de technicien supérieur agricole (BTSA).

\section{I.1.3. Un contexte également marqué par une importante réforme pédagogique}

La construction de ce secteur s'inscrit dans le cadre de la poursuite de la rénovation engagée avec le BTA. Il en reprend les idées maîtresses qui sont à la base de la conception des formations :

- la mise en place de toute formation professionnelle doit être précédée d'une analyse du métier, cela aboutit à l'élaboration d'un référentiel professionnel qui permet de décrire un ensemble de compétences à acquérir dans la formation ;

- l'offre de formation doit être structurée selon des champs professionnels. Il s'agit de rompre avec la référence étroite aux simples métiers et postes de travail pour tenir compte de ce qui fait l'environnement commun d'un ensemble de qualifications, d'une dimension collective de l'organisation du travail. On observe dans la pratique une complexification des qualifications par imbrication de différentes fonctions qui s'organisent autour d'une dominante. Ces qualifications sont de plus en plus référées à des compétences transversales de type organisationnelles.

Ainsi la problématique posée par la construction de nouvelles formations répondant aux évolutions du contexte s'est articulée dans l'enseignement agricole autour de deux questions :

- comment professionnaliser ces nouvelles fonctions, ces nouvelles activités, générées par de nouveaux besoins émanant de la société ? Il ne s'agit pas simplement d'enrichir, de modifier des contenus de formation mais de raisonner en termes de métiers: nouveaux métiers, évolution de métiers existants, et d'identifier la nature de nouvelles compétences à exercer.

- quels peuvent être les fondements d'une culture professionnelle commune à l'ensemble de ces métiers rassemblés dans un même secteur de formation?

\section{I.1.4. Les étapes de la construction de ce secteur}

En 1988, l'architecture des formations est définie par sept secteurs professionnels : production, transformation des produits agricoles et bio-industries, commercialisation, exploitation et 
aménagement de l'espace, tertiaire de bureau, tourisme et services en milieu rural, activités sportives équestres.

Le secteur qui nous intéresse s'appelle alors «exploitation et aménagement de l'espace ». Il propose des formations spécifiques au niveau $\mathrm{V}$ et au niveau III.

\begin{tabular}{|c|c|c|c|}
\hline $\begin{array}{c}\text { SECTEUR } \\
\text { PROFESSIONNEL }\end{array}$ & $\begin{array}{l}\text { CAPA } \\
\text { BEPA }\end{array}$ & $\begin{array}{c}\text { BTA BAC } \\
\text { PROFESSIONNEL }\end{array}$ & BTSA \\
\hline $\begin{array}{l}\text { Exploitation et } \\
\text { aménagement de } \\
\text { l'espace }\end{array}$ & $\begin{array}{l}\text { Exploitation et } \\
\text { aménagement de } \\
\text { l'espace }\end{array}$ & $\begin{array}{l}\text { BTA secteur } \\
\text { production }\end{array}$ & $\begin{array}{l}\text { Exploitation et gestion } \\
\text { forestière } \\
\text { Paysagisme } \\
\text { d'aménagement } \\
\text { Gestion des ressources } \\
\text { naturelles } \\
\text { Aménagement et } \\
\text { développement rural }\end{array}$ \\
\hline
\end{tabular}

Le niveau $\mathrm{V}$, comme dans tous les secteurs, est défini par un seul champ professionnel pouvant comporter différentes spécialités. Au niveau III une plus grande souplesse est envisagée, les champs d'activités sont plus diversifiés. Les orientations présentées dans ce tableau constituent une première ébauche de la définition de ce secteur.

La construction des référentiels débute en 1989, au moment où la rénovation aborde la réécriture des BEPA et CAPA. C'est donc à ce niveau que s'est amorcée une réflexion sur les métiers en émergence dans ce champ d'activité. Trois spécialités sont identifiées : travaux paysagers, travaux forestiers, entretien de l'espace rural. Les deux premières correspondent à la rénovation de qualifications existantes, la troisième représente le seul diplôme vraiment nouveau créé dans ce secteur. Les autres dérivent plus ou moins directement d'anciennes qualifications.

Pour définir le BEPA «Entretien de l'Espace Rural » (EER), il a fallu innover jusque dans la manière de construire un référentiel professionnel qui s'est largement inspirée des résultats d'opérations pilotes menées dans le cadre du dispositif «nouvelles qualifications $»^{2}$ mis en place à la fin des années 1980. Cette qualification tente de répondre à trois constats :

- un besoin important, émergeant dans de nombreuses zones rurales, concernant l'entretien des chemins, des bords de rivières, le débroussaillage et l'aménagement de zones susceptibles de constituer de nouveaux espaces de fréquentation. L'abandon de cet entretien réalisé jadis par les agriculteurs résulte en grande partie de la forte diminution des actifs en milieu rural ces dernières décennies ;

- la nécessité de développer de nouvelles compétences (par rapport à celles mises en œuvre par des entreprises forestières par exemple) à partir d'un changement qualitatif des pratiques

\footnotetext{
${ }^{2}$ L'originalité de cette démarche est de traiter conjointement les questions de l'évolution ou de la définition des postes de travail, et les modalités et les contenus des formations à dispenser. Ce dispositif s'intéressait prioritairement aux bas niveaux de qualifications très souvent oubliés dans les études prospectives. Les conclusions du groupe "environnement" regroupant différents sites, permettaient de construire la trame d'un référentiel.
} 
mises en œuvre c'est-à-dire une prise en compte des caractéristiques du milieu, et de la préservation des écosystèmes ;

- la nécessité de former des chefs d'équipes chargés d'encadrer une main-d'œuvre, non qualifiée, recrutée dans des chantiers de réinsertion, par exemple les « chantiers verts ».

Les contenus des différents référentiels sont ensuite définis progressivement entre 1989 et 1998, d'abord dans le cadre d'une réflexion sur les métiers menée en concertation avec les branches professionnelles concernées (référentiels professionnels), puis dans le cadre d'une réflexion pédagogique impliquant l'inspection (référentiels de formation).

Pour caractériser ce secteur, dans les textes d'accompagnement des référentiels, c'est sur la fonction remplie par les acteurs, sur la finalité de leur action que l'on insiste : l'amélioration du cadre de vie, le développement des loisirs, la protection et la conservation du patrimoine naturel. Dans certaines filières comme celle liée à la gestion forestière, une fonction domine : la production de bois, mais c'est au titre d'une plurifonctionnalité acquise récemment par ces milieux qu'elle a été rattachée à ce secteur. Le référentiel du BTSA aménagement forestier précise que la gestion forestière doit également prendre en compte une fonction sociale d'accueil du public, la considération de cet espace comme élément du paysage, du cadre de vie, une fonction de protection et de maintien de la biodiversité.

Les professionnels formés dans le cadre de ce secteur ont tous en commun, dans le projet affiché par les concepteurs, le fait de participer à travers la pratique de leur métier à une gestion de la qualité de l'environnement.

L'architecture finale de ce secteur est donc marquée par la coexistence de plusieurs familles de métiers relevant chacune d'une histoire, d'une culture, et d'une organisation professionnelle très différentes. La rénovation en structurant les formations par secteur les a rassemblées. Il nous semble important d'analyser ce qui fonde ce projet pédagogique et la mobilisation des savoirs sur lesquels il repose.

\section{I.2. Une analyse sociologique et épistémologique des nouveaux référentiels}

\section{I.2.1. Le cadre théorique et méthodologique mobilisé pour l'analyse des référentiels}

L'évolution d'un système éducatif ne peut être abordée comme une simple mise en conformité avec les évolutions sociales : «ce qui s'enseigne c'est moins la culture que cette part ou cette image idéalisée de la culture qui fait l'objet d'une approbation sociale et en constitue en quelque sorte la version autorisée, la face légitime » (Forquin, 1992). Par ailleurs l'école restructure ces matériaux culturels pour en faire des objets d'enseignement et en organiser l'apprentissage selon une logique didactique (Chevallard, 1985). L'approche sociologique des "curricula» à laquelle nous nous référons se différencie d'une sociologie de la connaissance dans la mesure où ses investigations échappent aux débats centrés sur la manière dont les savoirs sont produits, diffusés, légitimés au sein de la société selon des hypothèses constructivistes. Pour Forquin(1992), à la suite de socilogues comme Young et Bernstein, les enjeux sociaux de la construction d'un «curriculum » ne mettent pas 
en cause la valeur épistémologique, la fonction didactique des savoirs enseignés (Forquin, 1992). Il ne s'agit pas d'une construction sociale de savoirs, mais d'une structuration socialement pertinente des systèmes de transmission des savoirs que sont les «curricula ». Les savoirs sont principalement abordés sous l'angle de leur mode d'agencement au sein des programmes et cursus scolaires par exemple: est-il organisé en matière scolaire ou non, quelle est la part d'initiative laissée à l'enseignant en matière de choix et d'organisation des connaissances à transmettre ?

Dans notre recherche (Baradat-Bouillier, 1999), c'est le travail de traduction du projet affiché par l'institution en charge de l'enseignement agricole en «curricula» de formation que nous avons analysé en prenant en compte les problématiques propres à l'élaboration des "curricula » de l'enseignement technique et professionnel.

Ces filières de formation sont marquées par l'émergence, à partir des années 1980, de nouveaux outils d'orientation que sont les référentiels professionnels et les référentiels de formation. Les premiers décrivent le métier, les seconds traduisent les activités professionnelles en objectifs pédagogiques qui sont ensuite mis en relation avec différents types de savoirs: déclaratifs, procéduraux, pratiques. La logique de sélection et d'organisation de ces savoirs ne répond plus à un principe disciplinaire mais à la nécessité d'informer des catégories d'action. La pertinence d'un référentiel de formation professionnelle porte donc sur la lisibilité et sur le respect des logiques d'action propres à un champ technologique et à un métier. Mais sa fonction va au-delà. Le processus même d'élaboration du référentiel par une mise en texte de la pratique, conduit à formaliser non seulement les savoirs d'action mais également l'ensemble des démarches et des raisonnements qui informent et justifient ces actes (Rey, 2006). Ils construisent de fait une théorie de ces actes.

Nous avons considéré, pour notre analyse, que la pertinence d'un référentiel de formation professionnelle était fondée sur l'articulation entre les apprentissages technologiques et pratiques, et sur un ensemble d'éléments qui leur donnent sens, qui contribuent à doter l'individu d'un regard critique sur son environnement et à orienter son action.

Depuis la réforme de 1985, les référentiels de l'enseignement technique agricole sont structurés en modules pluridisciplinaires organisés selon une architecture globale relativement stable (BaradatBouillier, 1999; Bouillier, 2001). Une analyse de cette architecture, fondée sur le Brevet de Technicien Supérieur Agricole (BTSA), permet de mettre en évidence des liens entre les apprentissages relatifs aux processus technologiques et deux grands groupes de modules définissant des systèmes de signification des conduites professionnelles. Un premier groupe de modules porte sur l'apprentissage de raisonnements stratégiques qui permettent au technicien de situer son action au sein du système socio-économique dans lequel s'intègrent ses productions (l'exploitation agricole, les filières agroalimentaires et maintenant le territoire). Le deuxième groupe concerne l'enseignement général relié dans ces référentiels à des pratiques sociales. Nous avons identifié qu'il permettait d'articuler la dimension culturelle et la dimension technologique de l'activité professionnelle en faisant référence aux aspects symboliques et éthiques de l'acte technique et à ses conséquences sociales. Ce sont ces indicateurs que nous avons retenus pour structurer notre analyse des référentiels autour de trois questions : quelles sont les références culturelles qui donnent sens aux pratiques? Quelles références socio-économiques orientent les stratégies professionnelles ? Quelles sont les logiques d'action qui caractérisent les processus technologiques ? Notre analyse prend en compte non seulement le texte des référentiels, mais également l'ensemble des recommandations 
pédagogiques et documents d'accompagnements élaborés principalement par l'inspection pédagogique.

La lecture des modules transversaux (modules d'enseignement généraux et modules centrés sur l'approche socio-économique du secteur d'activité), dans une première étape, nous a permis de mettre en évidence les notions et concepts mobilisés par les concepteurs des référentiels.

Dans un deuxième temps, une mise en perspective de ces concepts vis à vis des débats scientifiques de l'époque nous a conduits à définir plus complètement le champ conceptuel qui permet de donner sens aux contenus de formation et aux pratiques des futurs professionnels dans les référentiels analysés.

\section{I.2.2. Lecture des référentiels rénovés}

\section{Les modules transversaux aux différentes spécialités}

Commençons par le niveau IV qui constitue le cœur de l'offre de formation de l'enseignement agricole. Le Brevet de Technicien Agricole (BTA) «Aménagement» créé en 1992 constitue une extension du BTA créé en 1985. Il se décline en trois spécialités : gestion et conduite des chantiers forestiers, gestion et conduite des chantiers paysagers, gestion de la faune sauvage.

Alors que dans le secteur production, avec lequel ce secteur est régulièrement mis en opposition, l'environnement professionnel est assimilé à l'environnement économique au sein duquel s'exerce l'activité, dans ce secteur, les logiques professionnelles se réfèrent à un modèle de gestion de l'environnement. Ainsi dans ce BTA, l'étude de l'exploitation agricole et d'une filière de production donnée font place à «l'analyse d'un système d'acteurs qui gèrent de manière concertée et négociée la qualité de notre cadre de vie ressenti comme bien commun, comme patrimoine » (commentaires pédagogiques p. 1). Il ressort du texte de présentation de ce secteur, une volonté de faire émerger des nouvelles pratiques professionnelles relevant d'un modèle de gestion patrimoniale de l'environnement et d'ouvrir le champ d'activité à de nouveaux espaces requalifiés par une demande sociale de qualité de l'environnement : forêts périurbaines, zones dégradées, friches industrielles, etc. (ibid., p. 1)

Les modules communs se réfèrent également à une culture du vivant systémique et fonctionnelle intégrant différents niveaux d'organisation : organisme, écosystème, biosphère.

$\mathrm{Au}$ niveau $\mathrm{V}$, qui représente un niveau de qualification professionnelle centré sur l'exécution de tâches techniques, le référentiel professionnel du BEPA «Entretien de l'espace rural » créé en 1991 définit l'aménagement comme une intervention sur le milieu pour le rendre propice à un usage social défini en termes de jouissance de la vue : qualité du paysage, du cadre de vie, ou en termes de fréquentation, pour préserver sa qualité écologique, pour prévenir des risques naturels : inondations, avalanches... dues à un manque d'entretien.

En réponse à cette définition les modules communs aux trois spécialités font une place importante à l'enseignement de la biologie et à la lecture de paysage. La connaissance du vivant est centrée sur l'écosystème en tant que système ouvert de relations. L'aménageur est présenté comme étant à l'origine d'une dynamique provoquée des écosystèmes (recommandations pédagogiques, p. 64). L'appréhension du paysage doit conduire à l'émergence de la notion de patrimoine comme bien commun dont l'altération entraîne une perte de reconnaissance et d'identité de ses acteurs. 
$\mathrm{Au}$ niveau III, ces fondements sont repris et déclinés de manière plus précise avec l'introduction d'une culture du paysage plus approfondie pour le BTSA «Aménagement paysager », cette culture devant conduire le futur technicien à «se situer comme partenaire d'une gestion négociée du cadre vie » (commentaires pédagogiques, p. 49).

Le forestier doit lui aussi situer sa pratique professionnelle dans une nouvelle perspective l'amenant à dépasser les logiques réglementaires et économiques qui orientent classiquement l'activité professionnelle forestière.

Le modèle de gestion patrimoniale est mis en avant pour chacune de ces deux spécialités ainsi qu'une approche systémique du végétal et du milieu d'intervention.

Le champ d'activité du technicien supérieur en "gestion et protection de la nature » renvoie à la définition problématique de la notion de nature et de protection de la nature. Que signifie la cohabitation des deux termes protection et gestion dans l'intitulé de la qualification ? Le référentiel professionnel définit le champ d'activité selon deux dimensions indissociables :

- le type d'espace sur lequel s'applique la gestion : les espaces naturels ;

- la réponse à une demande sociale de nature.

Les espaces naturels recouvrent ainsi des espaces non exploités par l'homme c'est-à-dire qui ne sont pas irréversiblement artificialisés ou qui présentent un intérêt écologique mais aussi les espace exploités à des fins de production mais qui sont perçus par la société comme des espaces de nature en raison notamment des paysages qu'ils donnent à voir.

Le technicien peut remplir deux fonctions : une fonction technique avec la mise en œuvre de plans de gestion intégrant une mise en valeur écologique, économique et culturelle du milieu, et une fonction d'animation qui vise la sensibilisation, l'éducation du public à la nature et à l'environnement, à l'interprétation des milieux. Dans cette fonction le technicien est présenté comme un «agent participant à l'éveil d'une conscience écologique » du public.

Là encore le technicien «qu'il gère la nature ou sa qualité ressentie comme patrimoine » (commentaires pédagogiques, p. 47), participe à « une gestion en bien commun de la qualité des espaces naturels, contractualisée, négociée entre tous les acteurs » (ibid., p. 46). Mais dans cette qualification, un module (D32), présenté comme la clé de voute de la formation, ajoute avec les deux modules centrés sur l'écologie «à la dimension humaine de la notion de patrimoine, son contenu scientifique » (ibid., p. 60). Cette connaissance scientifique est fondée sur une approche systémique et fonctionnelle du milieu vivant. Mais l'approche de l'expertise est ici nouvelle, ce ne sont pas des concepts scientifiques, leur contenu idéologique ou l'application réglementaire des logiques fondées sur ces concepts qui fondent l'action de ces techniciens mais la mobilisation de ces concepts dans le cadre d'une démarche de gestion patrimoniale situant l'enjeu de la gestion dans le système de relation entre un groupe d'acteurs et son territoire et non dans une vision figée de la préservation de la nature pour elle-même (ibid., p. 47).

\section{I.2.3. Le cadre conceptuel qui fonde la culture commune à l'ensemble des métiers de ce secteur}

\section{Les références culturelles qui donnent sens aux pratiques}

Une particularité de ce secteur de formation tient au caractère inédit des évolutions qui justifient sa création ainsi que l'évolution des contenus des différentes filières de formation. En effet ce ne sont ni des impératifs économiques ni des avancées technologiques qui constituent les moteurs du 
changement mais un ensemble d'évolutions culturelles qui conduisent à repenser nos liens à la nature et à la manière dont elle est exploitée.

Les concepts mobilisés pour fonder cette nouvelle culture professionnelle relèvent dans les textes analysés clairement d'une éthique environnementale. Ces concepts étaient, au moment de l'écriture des référentiels au début des années 1990, encore en construction au sein de la communauté scientifique et souvent mêlés à des argumentaires politiques au sein de débats, vifs à cette époque, sur la protection de l'environnement. Ils étaient encore peu diffusés au sein du monde scolaire et peu familiers pour les enseignants chargés de les enseigner.

Précisons le cadre conceptuel auquel les auteurs de ces modules font référence. Depuis le rapport Brundtland au sommet de Rio (1992) c'est autour du concept de développement durable que s'est construite une pensée légitime de l'environnement. Il traduit l'émergence d'une nouvelle éthique fondant l'action collective sur un ensemble de principes de précaution, de responsabilité et de solidarité entre nations et générations. Les fondements de cette éthique peuvent être reliés à des changements culturels importants concernant notre rapport à la nature et notre rapport au savoir (Baradat-Bouillier, 1999).

Pour caractériser les fondements de cette éthique environnementale nous avons analysé le contenu des conventions internationales qui ont progressivement jalonné la mise en place d'une gestion publique de l'environnement. Nous nous sommes focalisés également sur les grandes controverses publiques portant sur cette thématique, en particulier celle qui s'est développée autour de l'appel d'Heidelberg ${ }^{3}$ signé par 264 scientifiques dont 52 prix Nobel s'adressant « aux autorités responsables du destin de la planète » (ibid., p. 73). Ce texte a fonctionné comme un catalyseur générant de multiples réactions en chaîne que nous avons analysées pour identifier les différentes conceptions de la science et les différents systèmes de valeurs qui s'affrontaient autour de la problématique environnementale.

La reconnaissance de la nature en tant que patrimoine constitue l'un des fondements principaux de cette éthique de développement durable (Bouillier, 2001). L'écologie et la portée symbolique de ses concepts ont fortement contribué à fonder la nature, dont on sait que la perpétuation ne va pas de soi, comme un patrimoine. C'est sur cette symbolique patrimoniale, associée aux notions d'identité et de transmission, que peut se fonder l'appropriation collective d'une problématique environnementale dans le cadre de systèmes locaux de gestion de l'espace et l'élaboration d'une logique d'action cohérente.

Le concept de développement durable modifie également notre rapport au savoir. Les questions d'environnement mettent au défi les scientifiques confrontés à des phénomènes complexes et à la nécessité de produire des normes d'action. Elles offrent une voie privilégiée de développement de démarches de construction de connaissances relevant d'une méthodologie d'approche de la complexité : modélisation systémique, interdisciplinarité. Elles modifient le statut de l'expert, qui est non plus celui qui sait mais celui qui rend intelligible les questions et qui contribue à la mise en forme des connaissances dans des dispositifs locaux de gestion de l'environnement (BaradatBouillier, 1999).

Quel lien entre cette symbolique patrimoniale et les pratiques d'aménagement?

\footnotetext{
3 « Appel d'Heidelberg ». Le Monde du 3 juin 1992.
} 


\section{Les stratégies qui orientent les pratiques professionnelles}

On note que dans les référentiels de formation la notion de gestion supplante progressivement la notion d'aménagement. Dans le champ qui nous intéresse, il s'agit de la gestion d'espaces, de milieux, de ressources naturelles. Ces pratiques s'exercent au sein de ce que Mermet (1992) appelle des systèmes locaux de gestion de l'environnement ancrés dans des territoires. La gestion est ici définie comme l'ensemble des actions qu'un système d'acteurs exerce sur le milieu. Dans les référentiels de formation cette notion de gestion était souvent abordée sous un angle technique. Les nouveaux référentiels du secteur aménagement proposent une évolution vers de nouveaux modes gestion respectant une éthique environnementale, c'est-à-dire en gérant l'espace, les milieux, comme un patrimoine.

Dans ces projets de gestion deux formes de patrimoines coexistent (Donadieu, 1993) :

- la première concerne le milieu éco-biologique et renvoie à une gestion qui prend en compte les conséquences de l'action de l'homme sur les écosystèmes. Il s'agit de préserver la biodiversité du milieu ;

- la seconde est une représentation sociale et culturelle de l'espace : le paysage. Les projets d'aménagements ou de gestion contribuent à une « mise en paysage » de l'espace à partir d'un ensemble de codes culturels mettant en relation ces projets avec une demande sociale, un regard collectif traduisant les nouvelles attentes de la société.

La gestion de l'environnement repose sur une pratique collective puisqu'elle impose que la gestion soit coordonnée à une échelle pertinente au regard du fonctionnement écologique du milieu et de sa perception comme paysage. Cela fait apparaître la dimension politique du processus mentionnée dans les recommandations pédagogiques. L'enjeu est de substituer à une gestion effective résultant de l'action indépendante d'un groupe d'acteurs, une gestion intentionnelle, coordonnée sur un territoire, qui produise une qualité de l'environnement répondant aux attentes de la société. C'est à partir du modèle de gestion patrimoniale négociée proposé par Ollagnon $(1984 ; 1989)$ qu'il est possible d'analyser une évolution des pratiques de gestion de l'environnement relevant d'une logique de développement durable. C'est ce modèle auquel il est fait référence de manière constante dans les recommandations pédagogiques. Il est fondé sur l'idée qu'une véritable gestion préventive à long terme de l'environnement suppose que les acteurs situés à tous les niveaux de décision se sentent investis du problème à traiter et s'attachent à le résoudre. Il permet de dépasser une logique purement économique par l'introduction explicite d'une éthique: la transmission d'un patrimoine aux générations futures. Il permet également de dépasser une simple approche réglementaire en insistant sur la négociation et la recherche préalable d'une adhésion des différents acteurs aux finalités de cette gestion par une définition commune de la qualité souhaitée de l'environnement.

Comment cette culture commune s'harmonise-t-elle avec les logiques professionnelles des différentes spécialités de ce secteur?

\section{I.2.4. La dimension technologique et professionnelle des référentiels}

La notion d'aménagement évoque, dans son aspect technique, la volonté d'organiser l'espace, le milieu, pour le rendre propre à un usage, et dans sa dimension territoriale, l'idée d'une action collective visant le développement d'un territoire à la fois sous l'angle spatial et sous celui de l'exploitation des ressources. 
Dans les référentiels, les préconisations orientent les pratiques d'aménagement de l'espace vers une vision plus humble de l'action de l'homme et vers une vision moins sommaire du milieu physique considéré dans le passé comme un simple support pour des activités productives. Une approche plus écologique du milieu conduit à reconsidérer la pertinence d'aménagements destructeurs sur certains milieux, à mettre en œuvre des interventions plus sélectives tenant compte de la dynamique et des phénomènes de régulation des écosystèmes. Les techniques mises en œuvre sont souvent qualifiées de «douces », qu'il s'agisse de la taille des arbres, ou de la restauration de rivières, on cherche désormais à « accompagner » la nature à partir d'une perception globale de son fonctionnement.

Ainsi dans le BEPA «Entretien de l'Espace Rural», les deux modules qui définissent respectivement la démarche et les techniques il est préconisé d'adapter les choix techniques aux caractéristiques du site dans ses dimensions écologiques et esthétiques.

Au niveau du BTA, là encore les préconisations viennent renforcer les orientations définies dans les modules transversaux. Ainsi le module QA3 du BTA « Gestion de la Faune Sauvage » propose une démarche définie comme totalement nouvelle et innovante (p. 51) qui conduit à gérer l'habitat support de la faune sauvage comme un patrimoine dans lequel intervient d'autres logiques d'acteurs. Le BTA forestier propose de développer une approche écologique et systémique de la conduite des peuplements forestiers et de prendre en compte la diversité des fonctions remplies par la forêt dans le raisonnement du projet de gestion forestière, «dans tous les cas le forestier n'est qu'un partenaire d'une gestion concertée et négociée de la qualité de la forêt» (p. 68). Les évolutions du BTA «Aménagement Paysagers » sont moins nettes. Les propositions de développer une démarche globale et systémique de choix des végétaux, d'élaborer un plan de gestion sans isoler l'aménagement paysager de son environnement ne représentent pas en soi une innovation importante. L'évolution la plus significative porte sur un souhait de diversifier les milieux supports de la formation en «évitant de se cantonner aux exemples classiques : jardins privés, parcs et espaces verts publics en milieu urbain pour montrer certaines techniques propres à la réhabilitation ou l'aménagement de certains milieux : friches péri-urbaines, talus d'autoroute, bords de rivières... » (p. 89).

Au niveau des modules technologiques des différents BTSA, ces orientations sont approfondies et proposent au niveau du BTSA «Aménagement Paysager» de faire réfléchir le futur professionnel aux pratiques développées dans la filière paysage et de l'amener vers une nouvelle manière de considérer les espaces gérés par cette option non plus simplement comme une collection d'arbres remarquables, comme un décor, mais en tant que milieu vivant ayant une dynamique propre pour lequel se pose la question de sa durabilité.

La formation technologique du BTSA forestier est centrée sur une nouvelle façon d'élaborer le diagnostic à partie du duquel est élaboré le plan de gestion. L'analyse des potentialités et contraintes du milieu doivent prendre en compte les notions de biodiversité, de risques naturels et paysagers. Les objectifs de gestion doivent mettre en relation les aspects techniques et les aspects sociaux liés à l'utilisation du milieu.

Le BTSA «Gestions des Espaces Naturels » propose de former un spécialiste capable de conduire une démarche d'audit patrimonial au sein d'un système d'acteurs de la gestion d'un espace de nature. Sa capacité d'intervention sur les systèmes vivants repose sur un l'apprentissage d'un nouveau champ technologique encore en émergence à ce moment-là : le génie écologique.

\section{I.3. Eléments de synthèse sur la création de ce secteur à partir de l'analyse des référentiels}


Le projet fixé par le schéma prévisionnel national (1991-1993) était, rappelons-le «d'étendre les formations jusqu'à la prise en main de la gestion de notre environnement », l'orientation qui était donnée était de former de nouveaux spécialistes, de définir de nouveaux métiers. La réponse apportée à ce projet à travers la construction de ce secteur a été double :

- former des spécialistes de la gestion d'espaces considérés comme des espaces de nature, ou valorisés comme des patrimoines naturels, dotés d'un champ d'expertise nouveau : le génie écologique, à travers la création du BEPA entretien de l'espace rural, et l'ouverture de filières de BTSA gestion et protection de la nature ;

- sensibiliser l'ensemble des techniciens spécialisés dans la gestion de l'espace et des ressources naturelles à de nouvelles pratiques fondées sur une éthique environnementale.

Une lecture comparée de l'ensemble des référentiels professionnels et des référentiels de formation des diplômes des secteurs production et aménagement écrits entre 1985 et 1999 (Baradat-Bouillier, 1999) nous a permis de mettre en évidence un décalage entre l'évolution de la description des métiers de référence et l'évolution des contenus des formations. Les innovations pédagogiques précèdent une évolution de la conception des métiers. Ces conceptions sont conditionnées par le fonctionnement de la Commission Professionnelle Consultative (CPC) des métiers de l'agriculture, de l'agro-industrie et de l'espace rural, fortement marqué par les organisations professionnelles agricoles qui définissent à travers leurs positions une normalisation des métiers décrits, en particulier celui de chef d'exploitation agricole. Les référentiels de formation, censés découler de ces référentiels professionnels affichent en réalité une grande autonomie, notamment au niveau des modules généraux et des modules communs à un secteur professionnel. C'est à ce niveau, que les concepteurs, l'inspection et les bureaux de la DGER, peuvent introduire des éléments permettant de donner un sens nouveau à des pratiques dont l'évolution est conditionnée par les milieux professionnels.

Au niveau du secteur aménagement, la sensibilisation à une gestion patrimoniale de l'environnement représente certainement la dimension la plus originale et la plus innovante de cette culture commune. Le contenu des référentiels, et surtout, le texte très dense des commentaires pédagogiques traduisent une démarche très volontariste et très innovante, pas toujours bien comprise par les enseignants chargés de la mettre en œuvre.

En effet cette démarche, encore en construction dans les milieux scientifiques à l'époque de la création de ce secteur, s'harmonise plus ou moins bien avec la culture professionnelle propre à chacune des filières professionnelles.

Si elle est au cœur de la formation des techniciens spécialisés dans la gestion et la protection de la nature, elle demeure difficile à opérationnaliser dans les filières correspondant aux métiers de la forêt et des aménagements paysagers centrées sur une logique de gestion directe du milieu.

La réalité professionnelle que les élèves découvrent lors de leurs stages les place le plus souvent en situation de conseil technique vis-à-vis d'un gestionnaire exerçant un pouvoir exclusif sur un espace défini (propriétaire d'un boisement, propriétaire d'un jardin, service des espaces verts d'une collectivité). Leur domaine d'expertise est essentiellement technologique.

\section{Les évolutions du secteur aménagement lors de la dernière rénovation}




\section{1. Analyse du contexte social, culturel et scientifique dans lequel s'inscrit cette rénovation}

\section{II.1.1. L'influence de l'écologie}

Nous avons mis en évidence que dès sa création ce secteur de formation préconisait une gestion des espaces respectueuse de l'environnement. Le fait nouveau nous semble résider dans le développement même de l'écologie en tant que discipline et sa mobilisation pour fonder l'expertise de l'aménageur. En pénétrant le champ de l'aménagement des espaces, l'écologie redessine indiscutablement depuis quelques années le contour des pratiques professionnelles des aménageurs. Elle apporte une vision globale de l'impact de l'action d'aménagement sur le milieu, en proposant un ensemble de concepts permettant de qualifier la fragilité des écosystèmes, comme la notion de biodiversité en lien avec la gestion des milieux et le risque de leurs dégradations. Si les techniques de l'aménagement sont longtemps restées plus ou moins éloignées de la considération des relations entre les êtres vivants et leur environnement, force est de constater qu'elles y sont aujourd'hui de plus en plus associées. Le génie écologique en est l'illustration parfaite. Ainsi tel que le préconise les recommandations pédagogiques du référentiel de diplôme du BTSA «Gestion et Protection de la Nature »(GPN) rénové (p. 9), le génie écologique fait appel à «l'ensemble des moyens, des démarches et des techniques mobilisant les relations entre les êtres vivants et leur milieu au bénéfice mutuel de l'intégrité biologique des écosystèmes et de la qualité des services qu'ils rendent à l'homme ». La réalisation, la restauration ou la réhabilitation des espaces doit s'appuyer sur des processus naturels plutôt qu'en usant de techniques moins durables du génie civil. C'est une pratique innovante qui préoccupe et réunit différents acteurs de la filière aménagement des espaces naturels en cours de structuration (scientifiques, professionnels, enseignants) ${ }^{4}$.

Depuis la rénovation des diplômes de l'enseignement agricole la biologie et l'écologie sont intégrées dans la dimension technique des apprentissages. Cette évolution constitue une étape marquante. En effet, ce changement de configuration disciplinaire confère une forme «d'hybridation » entre les disciplines scientifiques et techniques qui révèlent de manière significative le changement plus global des types de relations société-nature et surtout la place et le rôle qu'occupe la notion développement dans l'orientation des pratiques professionnelles.

\section{II.1.2. Le développement d'une éducation au développement durable}

Nous faisions 1'hypothèse, dans notre analyse des référentiels datant des années 1990, que les concepts mobilisés pour définir une culture commune à l'ensemble des formations en aménagement relevaient d'une éthique de développement durable alors émergente. Depuis, les référentiels rénovés s'inscrivent de manière explicite dans un cadrage réglementaire ayant pour but de généraliser une éducation au Développement Durable (EDD) à l'ensemble du système éducatif.

Le concept de Développement Durable qui trouve son origine dans les conférences internationales organisées par l'ONU a progressivement migré vers l'école sous la forme d'un ensemble de

${ }^{4}$ "Former aux métiers de la biodiversité et du génie écologique dans l'enseignement agricole technique », $1^{\text {er }}$ séminaire d'échange et de travail entre enseignants, professionnels, chercheurs, du 22 au 24 janvier 2013 à Montpellier SupAgro. 
directives adressées à l'ensemble du système éducatif et révèle une intrication complexe du politique et de l'éducatif (Asloum \& Kalali, 2013).

La première circulaire (MEN, 2004), «Généralisation d'une éducation à l'environnement pour un développement durable (EEDD) », définit à la fois l'esprit et les contours de cette éducation au développement durable en précisant que la prise de conscience des questions environnementales, économiques, socioculturelles doit, sans catastrophisme mais avec lucidité, aider les élèves à mieux percevoir l'interdépendance des sociétés humaines avec l'ensemble du système planétaire et la nécessité pour tous d'adopter des comportements propices à la gestion durable de celui-ci ainsi qu'au développement d'une solidarité mondiale .

La circulaire du MEN (2007) reprise par la DGER (2007), lance une seconde phase de généralisation d'une «Éducation en vue du développement durable » dans les établissements d'enseignement et de formation professionnelle agricole et dans les établissements d'enseignement supérieur agronomique, agroalimentaire, vétérinaire et paysager, publics et privés sous contrat avec l'État. L'éducation au développement durable (EDD) vise à une large couverture par les programmes scolaires avec un ancrage dans les connaissances avec le souhait d'une plus grande contribution des disciplines. Cette circulaire affiche une volonté d'impliquer les établissements dans des démarches de projet.

La troisième circulaire (MEN, 2011) et une note service (DGER, 2010) parachèvent la généralisation. Les orientations sont réaffirmées dans une circulaire de 2011.

Ces différents textes de cadrages qui déterminent les orientations politiques et les instructions pour l'éducation relative à l'environnement puis au développement durable, ont précédé les dernières rénovations des diplômes de l'enseignement agricole et constituent de fait un nouveau cadre de référence pour les diplômes du secteur aménagement.

\section{II.1.3. L'influence de la didactique professionnelle}

Sans que les prescriptions s'y réfèrent explicitement, nous pensons que les évolutions observées dans la façon de concevoir les référentiels et de penser le processus de professionnalisation des élèves sont en partie marquées par les apports du courant de recherche en didactique professionnelle (Pastré, Mayen \& Vergnaud, 2006) porté notamment par l'équipe «développement professionnel et formation » de l'ENESAD. Des liens existent entre les services de la DGER chargées de l'écriture des référentiels et $1^{\prime} E N E S A D^{5}$, via le réseau des $\mathrm{DRIF}^{6}$, et plus récemment à travers les missions attribuées au Système National d'Appui (SNA). EDUTER ingénierie, qui coordonne l'écriture des référentiels, a contribué à organiser un séminaire de réflexion sur la méthodologie d'élaboration des référentiels de diplômes en juin 2007. Un guide d'écriture édité en Décembre 2009, par les DRIF, EDUTER, l'inspection et la sous-direction POFE constitue le cadre de référence actuel.

Le rapport de recherche «Introduire un référentiel de situations dans les référentiels de diplôme en BTSA » (Ferron, Humblot, Bazile \& Mayen, 2006), à partir d'une étude menée par l'équipe «Développement professionnel et formation »sur les métiers de la production aquacole constitue un point d'étape important dans cette évolution. La démarche expérimentale proposée (même si elle n'a

\footnotetext{
${ }^{5}$ Actuellement Agrosup-Dijon-EDUTER.

${ }^{6}$ Délégués Régionaux à l'Ingénierie de la Formation.
} 
pas été reprise intégralement dans les processus d'élaboration des référentiels) a contribué à faire évoluer les pratiques en cours.

La didactique professionnelle a développé ces dix dernières années un ensemble de concepts permettant de fonder l'ingénierie des formations à visée professionnelle sur une analyse du travail en situation. L'objectif est d'analyser quelles conceptualisations sont à l'œuvre lorsqu'un professionnel réalise une tâche et d'en produire une modélisation qui permette d'orienter l'organisation de son apprentissage dans un dispositif de formation. Un point important de la démarche porte sur l'importance accordée à la notion de situation (Mayen, 1999). En formation professionnelle ce ne sont pas les savoirs scientifiques et techniques qui sont premiers mais les situations de travail, complexes, globales, diverses et marquées par une grande variabilité. Il s'agit donc de partir de ces situations de travail et d'analyser ce qui fonde l'activité efficace des professionnels dans ce contexte. Cette analyse passe par la compréhension du «dialogue » que le sujet engage avec la situation. La didactique professionnelle, en s'appuyant sur la théorie de la conceptualisation dans l'action (Vergnaud, 1996), montre que l'action n'est pas la simple application à un contexte donné d'un ensemble de savoirs et de représentations existants. Toute action comporte une part irréductible de création que l'on peut assimiler à une forme opératoire de conceptualisation qui permet d'agir, qui oriente l'activité en sélectionnant certaines caractéristiques de la situation. Ceci explique l'importance accordée également en formation à des mises en situation permettant d'apprendre dans et par le travail.

Dans le cadre de la description d'un métier il s'agit de repérer les situations « emblématiques » du métier, celles que le futur professionnel doit absolument maitriser pour l'exercer. Chacune de ces situations doit ensuite être considérée dans sa "généricité » et être représentative d'une classe de situations professionnelles qui dans la réalité de l'exercice du métier et les aléas de l'action prendront des contours différents que le professionnel doit savoir gérer. A chaque classe de situations correspond une structure conceptuelle (une logique d'action) que le professionnel doit mobiliser et adapter pour agir efficacement.

On peut trouver dans ces différentes propositions le fondement de la nouvelle forme des référentiels.

\section{II.2. La traduction de ces évolutions dans les prescriptions}

\section{II.2.1. Une évolution marquée par la réforme de la voie professionnelle}

La forme selon laquelle se présentent les nouvelles prescriptions est marquée par la réforme de la voie professionnelle qui représente une mutation importante au sein du système éducatif français. Cette réforme vise une élévation générale du niveau de qualification des élèves sortant de la voie professionnelle, une augmentation de la poursuite d'étude ${ }^{7}$ et une meilleur insertion professionnelle en limitant les sorties du système éducatif sans diplôme.

\footnotetext{
${ }^{7}$ Le Baccalauréat Professionnel est un diplôme visant l'insertion professionnelle. La poursuite d'études est possible dans les formations supérieures courtes : BTSA, DUT.
} 
Depuis la rentrée 2009 et jusqu'en 2014 cette réforme, caractérisée par la mise en place d'un nouveau cursus en trois ans, se déploie sur l'ensemble des secteurs de formation. La certification du Brevet d'Etudes Professionnelles Agricole (BEPA) devient un diplôme intermédiaire et rénové ${ }^{8}$.

Cette nouvelle offre de parcours en trois années est structurée au niveau de la seconde en cinq champs professionnels ${ }^{9}$ regroupant chacun plusieurs spécialités de baccalauréat professionnel ${ }^{10}$ qui ont des éléments communs dans leurs référentiels d'activités professionnelles.

C'est ainsi qu'apparait à la rentrée scolaire 2009 le champ nature-jardin-paysage-forêt correspondant à la classe de seconde NJPF qui peut conduire à trois bac pro comme le montre le schéma ci-dessous qui présente l'ensemble de l'offre du secteur aménagement de la voie professionnelle au niveau $\mathrm{V}$ et IV.

\section{Les parcours de la voie professionnelle en aménagement}

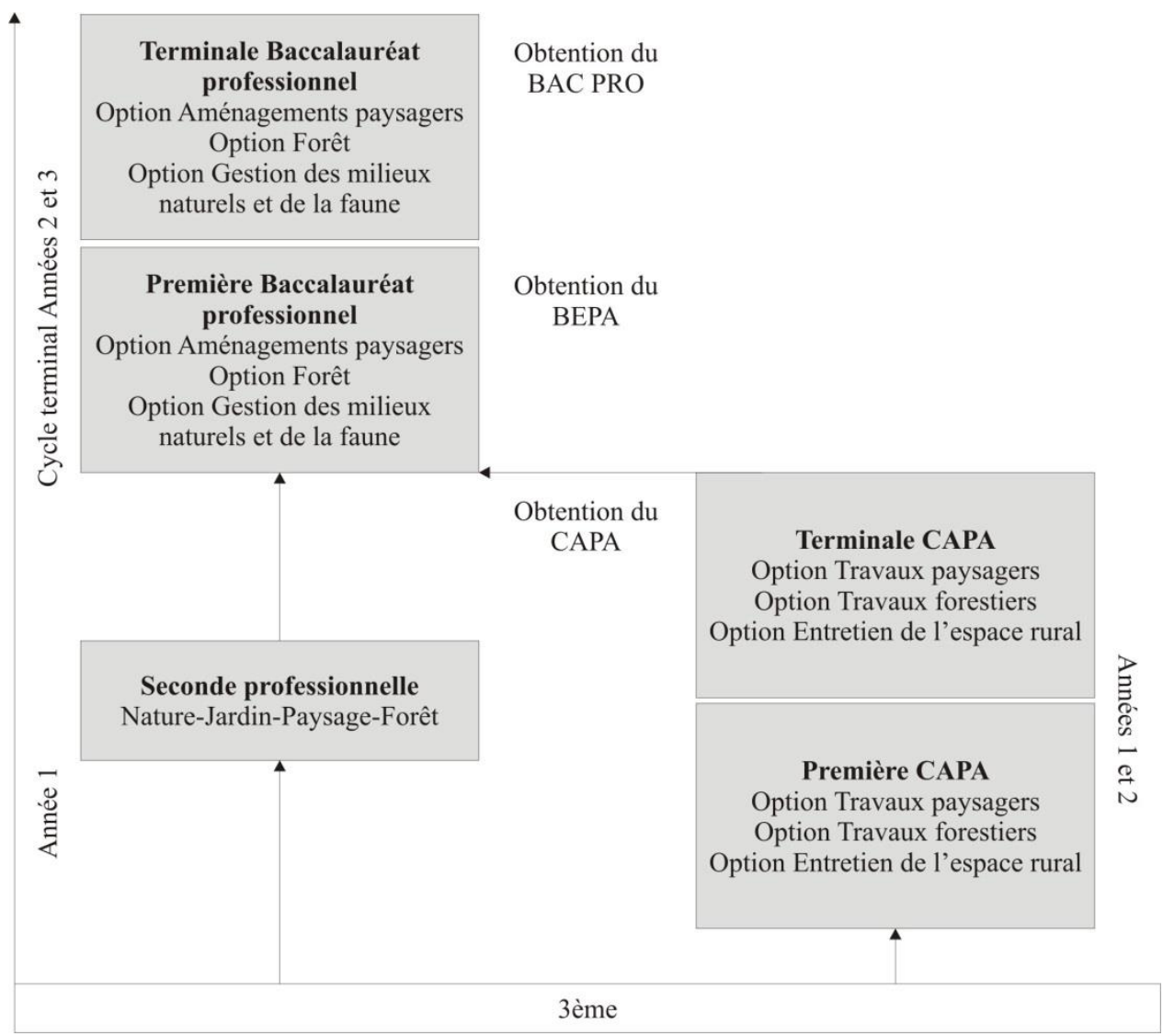

\footnotetext{
${ }^{8}$ Le cursus spécifique de formation vers le BEP est maintenu pour 4 spécialités. 12 spécialités sont progressivement rénovées (référentiels professionnels et référentiels de certification).

${ }^{9}$ Les champs professionnels de seconde constituent d'abord un moyen, pour des élèves plus ou moins déterminés dans le choix de leur spécialité de terminale, de confirmer ou d'infléchir leur choix à l'issue de la classe de seconde, ensuite un moyen d'aménagement de la carte des formations, permettant le regroupement en classe de seconde d'élèves qui préparent des spécialités de baccalauréat professionnel relevant du même champ.

${ }^{10}$ L'enseignement agricole compte 17 spécialités.
} 


\section{II.2.1. Des référentiels rénovés caractérisés par de nouveaux outils d'orientation de la professionnalité des formations}

Cela recouvre deux innovations principales :

- l'identification dans les référentiels professionnels de situations professionnelles significatives (SPS) ;

- l'introduction de référentiels de certification par capacité dans les nouveaux référentiels de diplômes.

\section{Les Situations Professionnelles Significatives (SPS)}

Leur définition résulte du travail d'enquête et d'analyse des métiers relativement fouillé réalisé par les DRIF qui dans le cadre de leur mission d'ingénierie, décrivent de manière détaillée l'activité des professionnels telle qu'elle se déroule sur le terrain (contextualisation des activités et ressources à mobiliser).

Ceci se traduit par le choix et l'écriture de situations professionnelles significatives (SPS) dans les nouveaux référentiels professionnels des filières de l'aménagement. Ces SPS représentent les situations clés, qui, si elles sont maitrisées par les titulaires des emplois visés par le diplôme, suffisent à rendre compte de l'ensemble des compétences mobilisées dans le travail. Elles définissent aussi le niveau de maitrise attendu pour un professionnel débutant, par conséquent le niveau d'exigence pour l'évaluation.

Les SPS sont utilisées par les équipes pédagogiques pour construire et mettre en œuvre des situations de formation qui se rapprochent le plus possible des situations professionnelles. Elles deviennent le socle des apprentissages requis pour l'exercice du métier.

Par ce biais, les prescriptions orientent la formation en aménagement vers une plus grande contextualisation de la formation incitant les enseignants à tenir compte du caractère complexe et incertain des réalités de terrain.

\section{L'évaluation par capacités}

La réforme de la voie professionnelle est également marquée par l'introduction de référentiels de certification qui s'ajoutent au référentiel professionnel et au référentiel de formation dans tous les référentiels de diplômes.

L'évaluation en aménagement ne repose plus sur la mesure d'objectifs à atteindre mais relève désormais d'une certification de capacités générales et professionnelles. Ce sont par exemple 8 capacités professionnelles qui sont évaluées en Bac Pro GMNF.

\begin{tabular}{|c|l|}
\hline $\begin{array}{c}\text { Capacités } \\
\text { professionnelles }\end{array}$ & \multicolumn{1}{c|}{ Intitulé } \\
\hline C5 & $\begin{array}{l}\text { Justifier des choix techniques d'intervention de génie écologique en fonction } \\
\text { d'enjeux sociaux, économique et environnementaux }\end{array}$ \\
\hline C6 & Analyser les conditions de mise en œuvre d'une action de génie écologique \\
\hline C7 & Communiquer en situation professionnelle \\
\hline C8 & $\begin{array}{l}\text { Conduire en sécurité le travail d'une équipe sur une intervention de génie } \\
\text { écologique dans une perspective de durabilité }\end{array}$ \\
\hline
\end{tabular}




\begin{tabular}{|l|l|}
\hline C9 & $\begin{array}{l}\text { Conduire en sécurité des travaux d'entretien d'espaces naturels et de } \\
\text { reconstitution d'écosystèmes }\end{array}$ \\
\hline C10 & Réaliser des actions de protection et de surveillance de l'environnement \\
\hline C11 & Mettre en œuvre des activités d'accueil du public et d'animation nature \\
\hline C12 & S'adapter à des enjeux professionnels particuliers \\
\hline
\end{tabular}

La capacité professionnelle sur laquelle reposent les nouveaux référentiels de certification est une aptitude à mobiliser et à combiner des ressources, autrement dit des connaissances pour réaliser une tâche dans une situation de travail simple ou complexe. Elle permet de mesurer un potentiel, une aptitude à résoudre un problème par la mobilisation de connaissances plurielles.

La capacité professionnelle dépasse donc la simple évaluation de connaissances ou d'habiletés pour ancrer la certification de la qualité professionnelle en aménagement dans un processus de contextualisation qui amène l'élève à réaliser des tâches qui demandent l'utilisation et l'application des savoirs appris dans un contexte de travail particulier. Ces situations contextualisées sont construites par les enseignants et permettent de relever des défis didactiques identifiés : comprendre, réussir, réaliser, s'adapter, etc. Ce sont les situations professionnelles significatives qui servent de références pour la construction des situations d'évaluation contextualisées dans lesquelles sont certifiées la ou les capacités professionnelles. Elles permettent de faire le lien entre les référentiels professionnels et de certification, entre la réalité des métiers et la mesure de l'aptitude à pouvoir les exercer.

\begin{tabular}{|c|l|l|}
\hline \multirow{2}{*}{$\begin{array}{c}\text { Champ de } \\
\text { compétences }\end{array}$} & \multicolumn{1}{|c|}{$\begin{array}{c}\text { Conduite d'opérations } \\
\text { techniques } \\
\text { d'aménagement paysager }\end{array}$} & \multicolumn{1}{|c|}{ Capacité professionnelle } \\
\cline { 2 - 3 } SPS & $\begin{array}{l}\text { SPS 5: implantation du } \\
\text { chantier } \\
\text { SPS 6: réalisation de } \\
\text { travaux d'aménagements } \\
\text { paysagers } \\
\text { SPS 7: gestion technique } \\
\text { et paysagère des } \\
\text { aménagements } \\
{[\ldots]}\end{array}$ & $\begin{array}{l}\text { C6. Assurer la mise en ouvre des techniques } \\
\text { appropriées à la situation d'aménagement, en } \\
\text { respectant la sécurité et l'environnement } \\
\text { décisions d'aménagement }\end{array}$ \\
\hline
\end{tabular}

SPS et capacités professionnelles. Référentiel professionnel du BTS « Aménagements paysagers »

L'évaluation par capacités s'inscrit dans une pédagogie de compétences. C'est par la confrontation à une pluralité de situations dans le travail réel que se constitue progressivement la compétence. Si la capacité peut s'apprendre dans le cadre d'une formation, la compétence nécessite une expérience de travail. La capacité professionnelle est une compétence en devenir.

Cet ensemble de changements contribue à renouveler le processus de professionnalisation aux métiers de l'aménagement qui nous intéresse ici et la nature des liens avec les milieux professionnels. 
Rappelons que l'orientation des formations de l'enseignement technique agricole est traditionnellement co-construite avec le monde professionnel dans le cadre de la commission professionnelle consultative (CPC)

La nouveauté dans cette réforme tient à une forme de mobilisation plus importante des professionnels au sein même du processus de formation et à une plus grande importance accordée aux séquences de formation en milieu professionnel et aux situations de formation proches de la réalité professionnelle.

\section{II.2.2. Un cadrage plus explicite des référentiels sur les questions environnementales}

Nous avons montré dans la première partie que dès les premières rénovations de diplômes au début des années 1990, le secteur aménagement avait déjà dans l'esprit et dans la lettre, intégré ces évolutions sans toutefois les associer de manière explicite au concept de développement durable ni à une démarche plus globale d'éducation au développement durable.

Notre expérience de formation d'enseignants nous a montré que les concepts nouveaux qui ont été introduits, peu articulés avec des contenus techniques au sein de référentiels exigeants n'ont pas toujours été compris. Leur mise en œuvre n'a pas été véritablement accompagnée de dispositifs et moyens pédagogiques permettant de prendre en charge la complexité de ces nouveaux contenus.

La notion de développement durable renvoie à des objets d'enseignement interdisciplinaires et la culture scolaire, la structuration des référentiels à partir des disciplines scolaires, évoluent lentement. Les changements souhaités lors de la création de ce secteur reposaient beaucoup sur le militantisme et la capacité des enseignants à traduire les orientations innovantes en situations de formations interdisciplinaires.

Les référentiels issus de la dernière rénovation sont plus concis, plus lisible et plus explicites. Les situations interdisciplinaires sont définies par une problématique, un nombre d'heures d'enseignement déclinées en différentes disciplines, des objectifs et des compétences (volet didactique). Des recommandations viennent préciser les modalités de mise en œuvre (volet pédagogique).

Dorénavant, le référentiel de formation est structuré selon deux grands groupes de modules :

- un premier groupe de module commun à l'ensemble des diplômes, porte sur la mobilisation d'une culture humaniste et sur la compréhension des faits économiques, sociaux et culturels. Ainsi des stages explicitement consacrés à une éducation au développement durable font leur apparition. La convergence des disciplines n'est plus laissée à la seule charge des enseignants, elle est désormais prescrite dans les différents modules. Par exemple dans le module professionnel, le M51 du BTSA aménagements paysagers «Contexte et déterminants des aménagements paysagers », le nombre de disciplines impliquées est conséquent. La biologie-écologie, les sciences économiques, sociale et de gestion, l'agronomie, l'histoire géographie et l'éducation socioculturelle sont directement associés à l'aménagement. Cette reconfiguration permet aux enseignants d'avoir une lecture plus explicite des rapprochements interdisciplinaires qui ne sont plus laissés à leur seule initiative. Des horaires spécifiques sont attribués à chaque discipline inscrite dans ce module. La prise en compte du contexte doit se construire sur la base de séances interdisciplinaires qui permettent de mobiliser et de faire converger les disciplines concernées. 
- un deuxième groupe de module concerne le domaine professionnel qui regroupe des modules spécifiques, ils visent à faire acquérir les connaissances scientifiques, technologiques, économiques et réglementaires du secteur professionnel. Les "situations professionnelles significatives » rattachées aux champs professionnels de l'aménagement qui servent de références pour la définition de situation de formation et de certification fixent la durabilité comme finalité de l'action.

\section{II.3. L'incitation à de nouvelles pratiques d'enseignement plus proches des réalités professionnelles : le chantier école}

Avec la rénovation de la voie professionnelle la séquence «chantier-école » apparait dans le référentiel de formation de la classe de seconde professionnelle. On parle de Travaux Pratiques Renforcés (TPR) dans les quatre autres champs professionnels (productions animales, productions végétales-agroéquipement, conseil vente, alimentation-bio-industrie-laboratoire). Le chantier-école constitue une situation de formation professionnalisante emblématique du secteur de l'aménagement qui se différencie des modalités de formation pratiques habituelles dans les établissements.

Les séances de travaux pratiques ponctuelles, hebdomadaires, régulières et de courtes durées (2 à 4 heures) sont déjà et depuis longtemps l'occasion de réaliser des exercices qui permettent l'acquisition des gestes et de postures liés à une tâche, la réalisation d'une opération technique élémentaire et ciblée : fosse de plantation, taille de haie, élagage, piégeage, prélèvements, etc.

Avec le chantier école la nouveauté ne tient donc pas à l'introduction d'un exercice pratique au service des apprentissages professionnels mais à la création d'un nouveau type de situation pédagogique complexe proche des réalités de terrain au sein de laquelle l'élève aura des décisions à prendre.

Dans ce contexte la tâche à réaliser ne relève plus uniquement d'une application isolée de connaissances et de savoir-faire techniques, l'élève doit construire et mettre en œuvre un raisonnement pour proposer une action adaptée aux spécificités d'un contexte.

Trois chantiers-écoles d'une semaine sont réalisés durant le parcours de formation menant à un Bac Pro aménagement permettant d'ancrer concrètement la formation professionnelle dans des pratiques relevant d'un développement durable, par exemple le fascinage et le tressage pour lutter contre l'érosion des berges d'un cours d'eau.

Ces situations d'apprentissage contextualisées permettent aussi de mieux travailler sur l'acquisition des capacités professionnelles telles que la $\mathrm{C} 7$ «communiquer en situation professionnelle ». Parce qu'il est collectif, le chantier-école est l'occasion de mettre les élèves en situation de travail en équipe. Ils s'aguerrissent concrètement à la gestion du groupe, à la coordination, à la concertation et au dialogue.

Si le chantier-école est une occasion de renforcer la qualité de la professionnalisation par la contextualisation des apprentissages, il est aussi un moyen de sortir de l'établissement, de déplacer le lieu de formation à l'extérieur, de s'ouvrir sur un territoire et créer du lien avec ses acteurs. Le chantier-école est un outil du lien établissement-territoire à construire. Il prend d'ailleurs davantage de sens pédagogique dès lors qu'il répond à la commande réelle d'une municipalité, d'une association ou d'une structure intercommunale (type syndicat 
intercommunal à vocations multiples, SIVOM) qui désire réhabiliter d'anciennes terrasses de cultures, restaurer les berges d'un cours d'eau, entretenir la végétation, etc. La mise en place de ce type de situation pédagogique vient évidemment questionner les pratiques des enseignants techniques pour qu'ils en valorisent toutes les potentialités notamment au regard de l'évaluation. Le chantier-école est aussi un outil de certification des capacités professionnelles.

\section{Conclusion}

Le secteur aménagement a été créé dans un contexte politique marqué par la nécessité de prendre en compte les préoccupations environnementales dans l'orientation des formations.

Il a joué un rôle particulier dans l'évolution globale du système de formation en concentrant le changement dans un premier temps sur la définition de nouveaux métiers «verts » renvoyant l'évolution de l'activité agricole et du conseil en agriculture à une réflexion ultérieure.

L'analyse des référentiels que nous avons conduite nous a permis de montrer que le caractère innovant de ce secteur, lors de sa création, était fondé sur une mise en relation des modules techniques et professionnels avec un ensemble de concepts émergeants destinés à leur donner sens et à doter les futurs professionnels d'un esprit critique sur leur environnement et les effets de leurs actions sur le milieu. L'aménageur est alors pensé par l'école comme un conseiller, plutôt qu'un technicien, chargé de mettre des connaissances à disposition de collectifs d'acteurs prenant en charge une gestion concertée de la qualité environnementale d'espaces ou de milieux au sein d'un territoire. Les modèles de gestion intégrée de l'environnement comme le modèle de gestion patrimoniale prônés par Ollagnon (1989) sont alors des concepts encore peu diffusés au sein même des milieux scientifiques.

Cette situation inédite mérite d'être soulignée dans ce qu'elle révèle du système éducatif agricole souvent présenté comme réactif, innovant et comme le fer de lance d'évolutions des milieux professionnels avec lesquels il est fortement lié.

Le volontarisme affiché par les concepteurs des référentiels n'a cependant que très progressivement influencé les pratiques d'enseignants confrontés à des référentiels exigeants, souvent peu compatibles avec des mises en situations professionnelles des élèves.

Il a manqué à cette première étape un «temps didactique » permettant à la sphère de la formation de traduire en objets d'enseignements les nouveaux concepts scientifiques permettant de les rendre compréhensibles par les enseignants et compatibles avec l'organisation pédagogique des établissements.

C'est une nouvelle approche du processus de professionnalisation des élèves conjuguée aux évolutions des milieux professionnels de l'aménagement qui caractérisent les changements issus des dernières rénovations.

Ces changements viennent des apports au monde de l'enseignement de nouvelles disciplines, comme l'ergonomie et la psychologie mobilisées dans l'analyse du travail. Reprises par le courant de recherche en didactique professionnelle pour fonder une nouvelle ingénierie de la formation, elles ont influencé la manière de concevoir les référentiels. 
Plus que de nouveaux contenus, ce sont donc de nouveaux outils d'orientation des formations comme les situations professionnelles significatives et le développement de chantiers écoles qui caractérisent les dernières évolutions qui permettent de contextualiser l'enseignement pour donner sens aux apprentissages. L'évaluation par capacité amène l'élève à produire une analyse de ces situations et à justifier son action en mobilisant les connaissances appropriées.

Enfin, dans le sillage d'une éducation au développement durable la manière de prescrire l'interdisciplinarité a également évolué. Des situations, identifiées dans les référentiels mobilisent explicitement un ensemble de disciplines au service d'une vision systémique du milieu et de l'acte d'aménager. L'écologie occupe une place centrale dans cet ensemble. 


\section{Bibliographie}

ASLOUM, N., \& KALALI, F. (2013). Repères historico critiques de l'évolution des curriculums prescrits de l'enseignement Agricole et de l'Education Nationale. Cas de l'éducation au développement durable. Penser l'éducation, hors-série, 449-466.

BARADAT-BOUILLIER, M.-H. (1999). L'introduction des problématiques environnementales dans l'enseignement technique agricole français. Thèse de doctorat, Université Toulouse I.

BOUILLIER, M.-H. (2001). Former aux métiers de l'environnement. Les orientations de l'enseignement technique agricole français. Education permanente, 148, 143-152.

CHEVALLARD, Y. (1985). La transposition didactique: du savoir savant au savoir enseigné. Paris : La pensée sauvage.

DONADIEU, P. (1993). Du désir de patrimoine aux territoires de projets. Thèse de doctorat, Université de Jussieu-Paris VII.

FORQUIN, J.-C. (1992). Ecole et culture. Le point de vue des sociologues britanniques. Revue française de pédagogie, 91, 123-125.

FERRON, O., HUMBLOT, J.-P., BAZILE, J., \& MAYEN, P. (2006). Introduire un référentiel de situations dans les référentiels de diplôme en BTS. Rapport de recherche remis à la DGER.

FOUREZ, G. (1996). Alphabétisation scientifique et technique. Essai sur les finalités de l'enseignement des sciences. Revue française de pédagogie, 115, 133-134.

MAYEN, P. (1999). Des situations potentielles de développement. Education permanente, 139, 101114.

MERMET, L. (1992). Stratégies pour la gestion de l'environnement. La nature comme jeu de société. Paris : L'Harmattan.

OLLAGNON, H. (1984). Acteurs et patrimoine dans la gestion de la qualité des milieux naturels. Aménagement et nature, 74, 1-4.

OLLAGNON, H. (1989). Une approche patrimoniale de la qualité du milieu naturel. Du rural à l'environnement. La question de la nature aujourd'hui. Paris : L'Harmattan.

PASTRE, P., MAYEN, P., \& VERGNAUD, G. (2006). La didactique professionnelle. Revue française de pédagogie, 154, 145-198.

REY, B. (2006). Les compétences professionnelles et le curriculum des réalités conciliables. Savoirs professionnels et curriculum de formation. Laval : Les Presses de L’université Laval. 
TROTTIER, C. (1987). La nouvelle sociologie de l'éducation en Grande Bretagne : un mouvement de pensée en voie de dissolution ? Revue française de pédagogie, 78, 5-20.

VERGNAUD, G. (1996). Au fond de l'action, la conceptualisation. Savoirs théoriques et savoirs d'action. Paris : PUF. 275-292.

\section{$\underline{\text { Textes concernant les prescriptions }}$}

MEN. (2004). Généralisation d'une éducation à l'environnement pour un développement durable. Circulaire ${ }^{\circ} 2004-110,8$ juillet 2004.

MEN. (2007). Education au développement durable, $2^{e}$ phase de généralisation de l'éducation au développement durable (EDD). Bulletin Officiel, 5 avril 2007.

DGER. (2007). Education en vue du Développement Durable (EDD) dans les établissements d'enseignement et de formation professionnelle agricole. Circulaire C2007-2015, 12 septembre 2007.

MAAF-DGER. (2009). Référentiel de formation Classe de seconde professionnelle "nature-jardinspaysage-forêt ».

IEA. (2009). Document d'accompagnement thématique "thème mise en auvre d'un chantier école».

DGER. (2010). Note de service organisation de la rentrée scolaire 2011. N2010-2165

24 novembre 2010.

MEN. (2011). Éducation au développement durable. Troisième phase de généralisation. Circulaire $\mathrm{n}^{\circ}$ 2011-186, 24 octobre 2011.

MAAF - DGER. (2011). Référentiel de diplôme Baccalauréat professionnel "aménagements paysagers».

MAAF - DGER. (2011). Référentiel de diplôme Baccalauréat professionnel "gestion des milieux naturels et de la faune ». 